\title{
Therapeutic Ionizing Radiation to Head History Absent
}

National Cancer Institute

\section{Source}

National Cancer Institute. Therapeutic lonizing Radiation to Head History Absent. NCI

Thesaurus. Code C160163.

There is no history of the individual receiving therapeutic ionizing radiation to the head. 